\title{
Cross Beam STAP for Nonstationary Clutter Suppression in Airborne Radar
}

\author{
Yongliang Wang, Keqing Duan, and Wenchong Xie \\ Key Research Laboratory, Wuhan Early Warning Academy, Wuhan 430019, China \\ Correspondence should be addressed to Keqing Duan; duankeqing@yahoo.cn
}

Received 4 April 2013; Accepted 30 August 2013

Academic Editor: Hang $\mathrm{Hu}$

Copyright (C) 2013 Yongliang Wang et al. This is an open access article distributed under the Creative Commons Attribution License, which permits unrestricted use, distribution, and reproduction in any medium, provided the original work is properly cited.

A novel space-time adaptive processing (STAP) method for nonstationary clutter suppression is proposed. The developed method forms a multibeam along the cross line to participate in adaptive processing, which sufficiently utilizes the spatial information both in azimuth and elevation and guarantees the least system degrees of freedom (DOFs). The characteristics of this structure help to suppress the short-range clutter which is the primary component of nonstationary clutter. Therefore, this method provides favorable clutter suppression performance when clutter range dependence exists. Approach analysis and simulation results are given to demonstrate the effectiveness of the method.

\section{Introduction}

Space-time adaptive processing (STAP) can achieve perfect clutter suppression performance in stationary clutter circumstance [1]. However, for non-side looking airborne radar (non-SLAR) (i.e., the radar with inclined side looking array and forward looking array), the clutter is range dependent or nonstationary, especially at short range [2]. In other words, the clutter is mostly nonstationary in practice because of detecting in all directions. Nonstationary clutter can result in the degraded performance of the adaptive processor compared to theoretical predictions.

So far, much effort has been put into this problem. The multiple-PRFs STAP scheme can relieve the blind regions induced by non-side looking and range ambiguity [3]. The main contribution of this attempt is just for solving the range ambiguity problem and not resolving the short-range clutter problem in essence. Compensation methods [4-6] work well when range ambiguity does not exist. Once the radar works at the medium or high pulse repletion frequency (PRF), it cannot identically compensate the ambiguity clutter. In fact, the short-range clutter, induced from the array elevation sidelobe, is the dominant reason for the clutter nonstationarity. The 3D STAP [7] with elevation elements or subarrays can cancel the short-range clutter effectively in theory. Unfortunately, it is hard to get enough independent identically distributed (IID) sample data and requires more computation load because of its large system DOFs. Subarray synthesis algorithm with prefiltering in elevation [8] can effectively suppress the short-range clutter when the element errors do not exist. However, the array antenna errors are inevitable in practice, thereby significantly degrading the performance. In this paper, a STAP method based on cross beam forming is presented in which the auxiliary beams are reasonably chosen in elevation and azimuth to cancel the clutter induced by elevation and azimuth sidelobes.

This paper is organized as follows. In Section 2, the principle of the cross beam STAP method is introduced. Section 3 shows the approach analysis. Simulation results are shown in Section 4, and Section 5 gives the conclusions.

\section{Clutter Characteristics for Non-SLAR}

In this section, the clutter math model is described and the clutter characteristics for non-SLAR are analyzed. Note that range ambiguity is considered in this model. As shown in Figure 1, the airplane flies along $X$-axis with velocity $V$, the $\theta_{p}$ is the angle between the array axes and flying direction, $\theta_{a}$ 


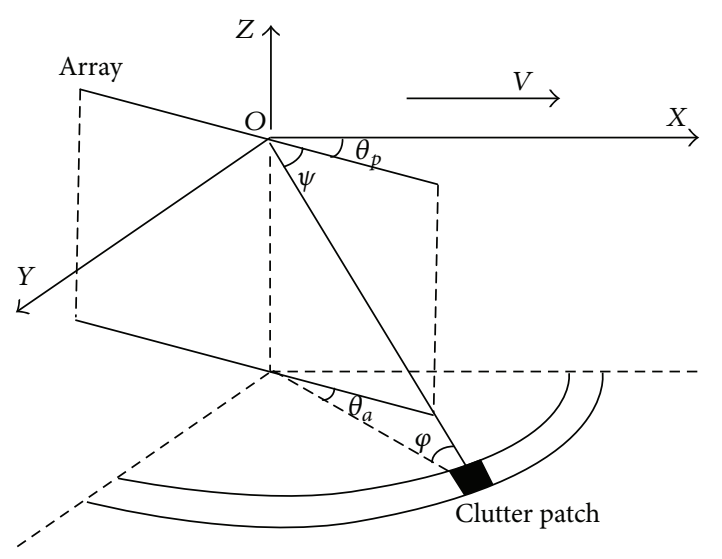

FIGURE 1: Geometry of non-SLAR.

and $\varphi$ are the azimuth angle and elevation angle of the clutter patch, respectively, and $\Psi$ denotes the cone angle.

The normalized Doppler frequency of the clutter patch can be described as follows:

$$
\begin{aligned}
\bar{f}_{d} & =\frac{2 f_{d}}{f_{r}}=\frac{4 V}{\lambda f_{r}} \cos \left(\theta_{a}+\theta_{p}\right) \cos (\varphi) \\
& =\frac{4 V}{\lambda f_{r}}\left(\cos \psi \cos \theta_{p}-\sin \theta_{p} \sqrt{\cos ^{2} \varphi-\cos ^{2} \psi}\right),
\end{aligned}
$$

where $\lambda$ is the wavelength, $f_{d}$ and $f_{r}$ denote the Doppler frequency and the PRF, respectively. From (1) we see that the Doppler frequency of the clutter patch varies with the cone cosine when $\theta_{p}$ is nonzero (i.e., the situation of non-SLAR). In other words, the distribution of clutter spectrum varies with range.

The clutter spectrum characteristic of a forward-looking planar array in azimuth-Doppler plane and range-Doppler plane is shown in Figures 2 and 3, respectively. The scenario parameters are given in Section 5. In Figure 2, we see that the clutter is comprised of three parts which are named $X_{1}, X_{2}$, and $X_{3}$. According to the conventional planar array pattern, we can conclude that $X_{1}, X_{2}$, and $X_{3}$ are, respectively, induced from elevation sidelobe, azimuth main lobe, and azimuth sidelobe. From Figure 3, we can see that the Doppler frequency of short-range clutter $X_{1}$ severely varies with range.

\section{Principle of the Cross Beam STAP}

A schematic of the proposed STAP architecture is shown in Figure 4 . An $M$ row by $N$ column vertical rectangular array is considered. The first stage is beam forming involving $M N$ digitized spatial elements or subarray channels. This results in a total of $P+Q-1$ beams which include main beam, $Q-1$ auxiliary beams along elevation with the azimuth main lobe, and $P-1$ auxiliary beams along azimuth with the elevation main lobe. One thing to be noted is that $P$ or $Q$ is always an odd number. After Digital Fourier Transform (DFT) for the data received from each beam, adaptive processing is applied for clutter suppression.

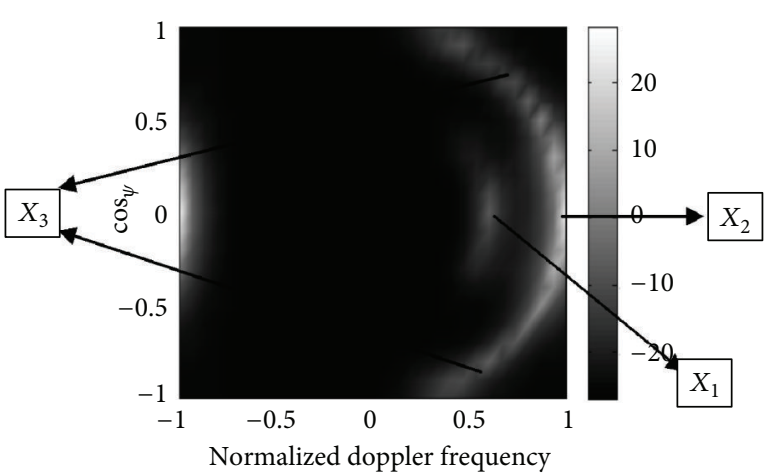

FIGURE 2: Clutter spectrum distribution of forward-looking planar array in azimuth-Doppler plane.

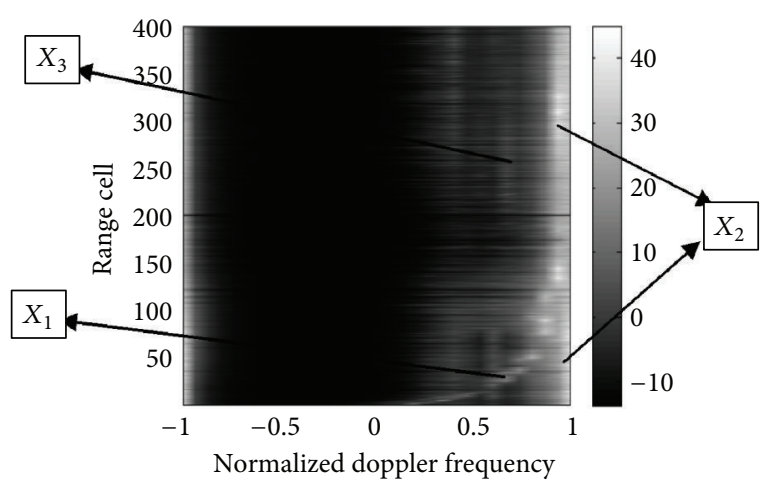

FIGURE 3: Clutter spectrum distribution of forward-looking planar array in range-Doppler plane.

The received data can be reshaped into an $M N \times K$ data cube $\mathbf{X}$. Let $\mathbf{X}_{M N} \in C^{1 \times K}$ denote the echo signals received by the $m$ th element or subarray channel in elevation and the $n$th element or subarray channel in azimuth, and let $K$ be the pulse number in one coherent pulse interval. The multiple beams can be obtained by

$$
\mathbf{A}=\mathbf{T}_{a}^{H} \mathbf{X}, \quad \mathbf{E}=\mathbf{T}_{e}^{H} \mathbf{X},
$$

where $(\cdot)^{H}$ denotes the conjugate transpose, $\mathbf{T}_{a} \in C^{M N \times P}$ and $\mathbf{T}_{e} \in C^{M N \times Q}$ are the azimuth and elevation beam transformation matrix, respectively. The $p$ th column of $\mathbf{T}_{a}$ and the $q$ th column of $\mathbf{T}_{e}$ have the following forms:

$$
\begin{aligned}
\mathbf{T}_{a p}= & {\left[\mathbf{S}_{s e 0}, \mathbf{S}_{s e 0} e^{j \pi \cos \theta_{p} \cos \varphi_{0}}, \ldots,\right.} \\
& \left.\mathbf{S}_{s e 0} e^{j \pi(N-1) \cos \theta_{p} \cos \varphi_{0}}\right]^{T}, \quad p=1,2, \ldots, P, \\
\mathbf{T}_{e q}= & {\left[\mathbf{S}_{s e q}, \mathbf{S}_{s e q} e^{j \pi \cos \theta_{0} \cos \varphi_{q}}, \ldots,\right.} \\
& \left.\mathbf{S}_{s e q} e^{j \pi(N-1) \cos \theta_{0} \cos \varphi_{q}}\right]^{T}, \quad q=1,2, \ldots, Q,
\end{aligned}
$$

where $(\cdot)^{T}$ denotes the transpose, $\theta_{p}(p=1,2, \ldots, P)$ and $\varphi_{q}$ $(q=1,2, \ldots, Q)$ are the angle of beams in azimuth and the angle of beams in elevation, respectively, and $\theta_{0}=\theta_{(p+1) / 2}$ 


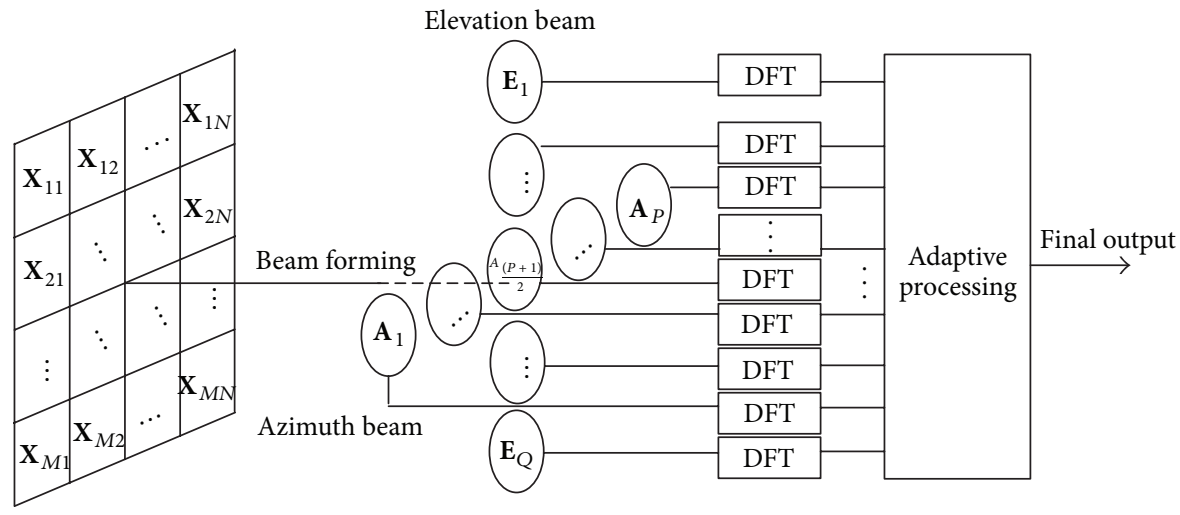

FIGURE 4: Schematic of the cross beam STAP method.

and $\varphi_{0}=\varphi_{(q+1) / 2}$ are the azimuth angle and elevation angle of the main lobe. $\mathbf{S}_{s e 0}$ and $\mathbf{S}_{s e q}$ are defined as follows:

$$
\begin{aligned}
& \mathbf{S}_{s e 0}=\left[1, e^{j \pi \sin \varphi_{0}}, \ldots, e^{j \pi(M-1) \sin \varphi_{0}}\right], \\
& \mathbf{S}_{\text {seq }}=\left[1, e^{j \pi \sin \varphi_{q}}, \ldots, e^{j \pi(M-1) \sin \varphi_{q}}\right] .
\end{aligned}
$$

Then the transformation matrix for cross beams can be written as

$$
\mathbf{T}_{s}=\left[\mathbf{T}_{a}, \mathbf{T}_{e}\left(1,2, \ldots, \frac{Q-1}{2}, \frac{Q+3}{2}, \ldots, Q\right)\right] .
$$

Let $\mathbf{T} \in C^{M N K \times(P+Q-1) L}$ be the transformation matrix in space and time domain. Then it has the following form:

$$
\mathbf{T}=\mathbf{T}_{t} \otimes \mathbf{T}_{s}
$$

where $L$ is the dimension after dimension reducing in time domain, $\otimes$ denotes the Kronecker product, and $\mathbf{T}_{t} \in C^{K \times L}$ is the dimension-reduced matrix in time domain and often transforms the pulse data to several adjacent Doppler bins in most conventional STAP algorithms.

After transforming the received data $\mathbf{X}$ to beam-Doppler domain, we can get the optimum weight by solve the following optimization problem:

$$
\begin{array}{ll}
\min & \mathbf{W}^{H}\left(\mathbf{T}^{H} \mathbf{R} \mathbf{T}\right) \mathbf{W}, \\
\text { s.t. } & \mathbf{W}^{H}\left[\mathbf{T}^{H}\left(\mathbf{S}_{t} \otimes \mathbf{S}_{s}\right)\right]=1,
\end{array}
$$

where $\mathbf{R}=E\left[\operatorname{Vec}(\mathbf{X}) \operatorname{Vec}(\mathbf{X})^{H}\right], \operatorname{Vec}(\cdot)$ denotes the matrices operation that stacks the matrix under each other to form a column vector, and $\mathbf{S}_{t}$ and $\mathbf{S}_{s}$ are the time domain and space domain steering vectors which can be represented by

$$
\begin{gathered}
\mathbf{S}_{t}=\left[1, e^{j 2 \pi f_{d} / f_{r}}, \ldots, e^{j 2 \pi(K-1) f_{d} / f_{r}}\right]^{T}, \\
\mathbf{S}_{s}=\left[\mathbf{S}_{s e 0}, \mathbf{S}_{s e 0} e^{j \pi \cos \theta_{0} \cos \varphi_{0}}, \ldots, \mathbf{S}_{s e 0} e^{j \pi(N-1) \cos \theta_{0} \cos \varphi_{0}}\right]^{T} .
\end{gathered}
$$

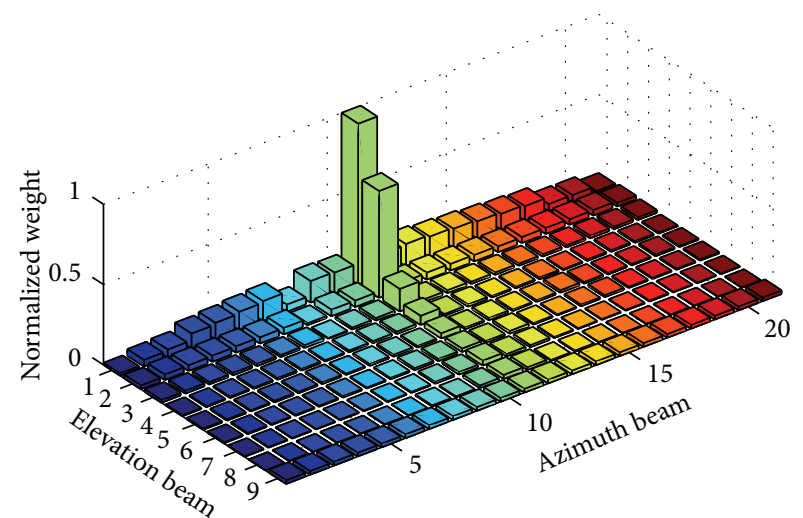

FIGURE 5: Adaptive weight normalized amplitude of the multibeams at the 28th Doppler bin.

\section{Approach Analysis}

For STAP in non-SLAR, the more system degrees of freedom (DOFs) in elevation, the better the nonstationary clutter suppression performance can be achieved $[7,8]$. However, the added elements or subarrays in elevation lead to the huge requirements for IID samples and computation load. The 3D STAP [7] is a typical example for that. Fortunately, it can be more flexible and effective to utilize the information in elevation if the data are transformed into beam domain. Figure 5 shows the normalized amplitude of the adaptive weight in space domain at the 28th Doppler bin when all the received data are transformed to multibeams. The clutter at the 28th Doppler bin is comprised of short-range clutter and azimuth sidelobe clutter. It can be seen from Figure 5 that the adaptive weight amplitude is large in the cross lines aiming at the elevation and azimuth main beam region, and small in the other position. It further demonstrates that the selection strategy of the cross beams is reasonable for nonstationary clutter suppression.

Figure 6 shows the eigenspectrum of the clutter at the 28th Doppler bin. The scenario parameters are described in the next part. From Figure 6 we can see that the eigenvalue number of cross beams is the least and the eigenvalue number 


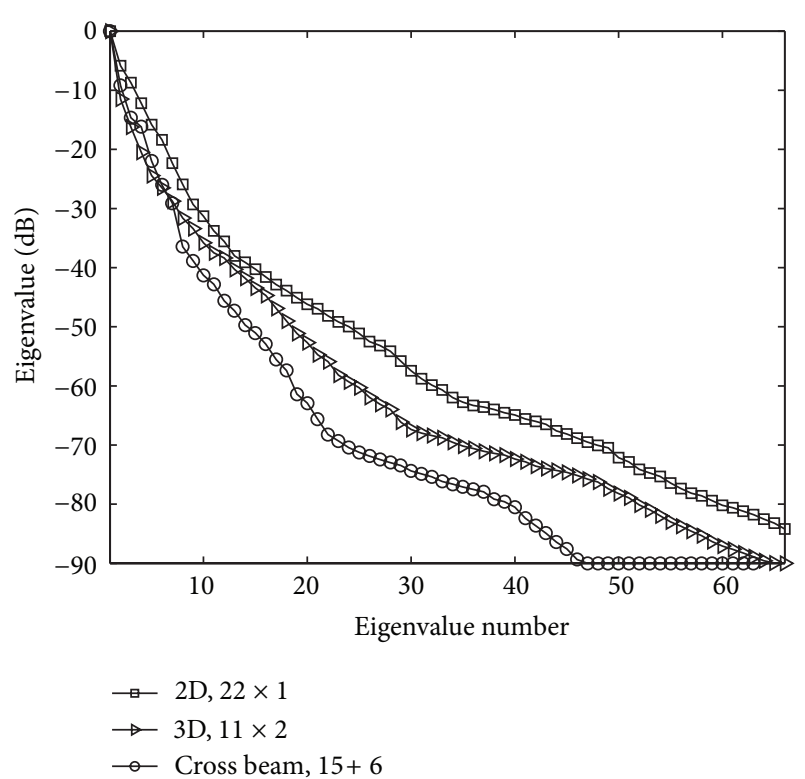

FIGURE 6: Eigenspectrum of clutter at the 28th Doppler bin.

of synthesized channels is the most. This result indicates that there are more redundant system DOFs to suppress clutter [9] for the new method compared with conventional 2D and 3D STAP method.

\section{Simulation Results}

A 220-element forward-looking planar array steered to $0^{\circ}$ in azimuth and $3^{\circ}$ in elevation is used, in which 22 elements are in azimuth and 10 elements are in elevation. Three correlative STAP methods are considered in this simulation for comparison. The typical 2D STAP with synthesized 22 spatial channels in azimuth is firstly considered. The second is the 3D STAP method with synthesized $11 \times 2$ spatial channels in azimuth and elevation. The recent STAP method based on elevation spatial prefiltering is also investigated in ideal and error conditions, respectively. The platform height is $8000 \mathrm{~m}$. The platform velocity is chosen so that there are no Doppler ambiguities. The pulse number and Doppler bins are both 32 and the short-range clutter distributed at the 24th to 30th Doppler bins. The number of Doppler bins for STAP is selected as 3 . The range cell under test is chosen as 50 . The numbers of formed beams are 21 and 9 in azimuth and elevation, and $P$ and $Q$ are, respectively, chosen as 15 and 7.

Figure 7 shows the signal-to-clutter-plus-noise ratio (SCNR) loss (i.e., loss relative to the clutter-free case) against Doppler frequency for a target located at the look direction when there are no errors in array. From Figure 7 we can see that the short-range clutter suppression performance of $3 \mathrm{D}$ STAP is better than that of the 2D STAP and worse than elevation prefiltering and cross beam STAP method when array errors are ignored. In this condition, the performance of cross beam STAP is close to the elevation prefiltering STAP method.

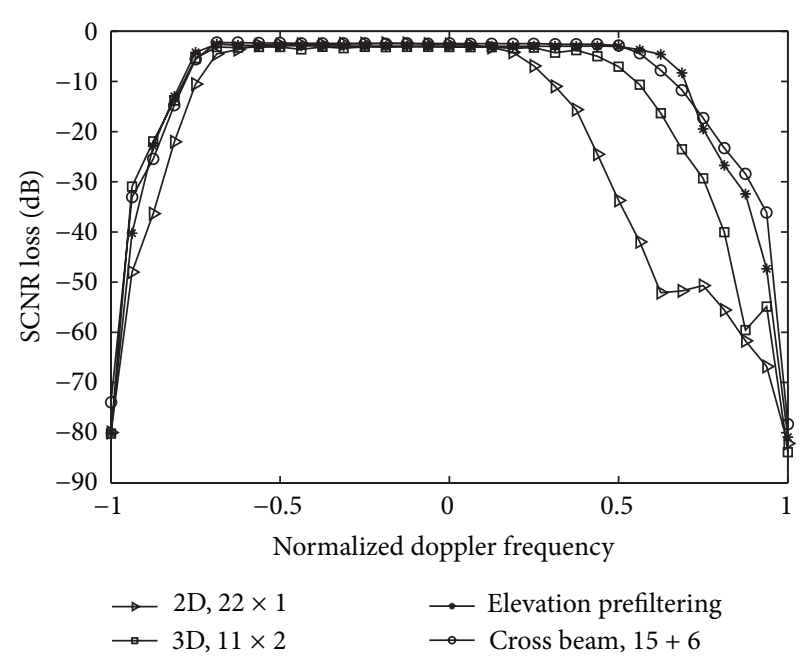

FIGURE 7: SCNR loss comparison of 2D STAP, 3D STAP, elevation prefiltering STAP, and cross beam STAP (no errors).

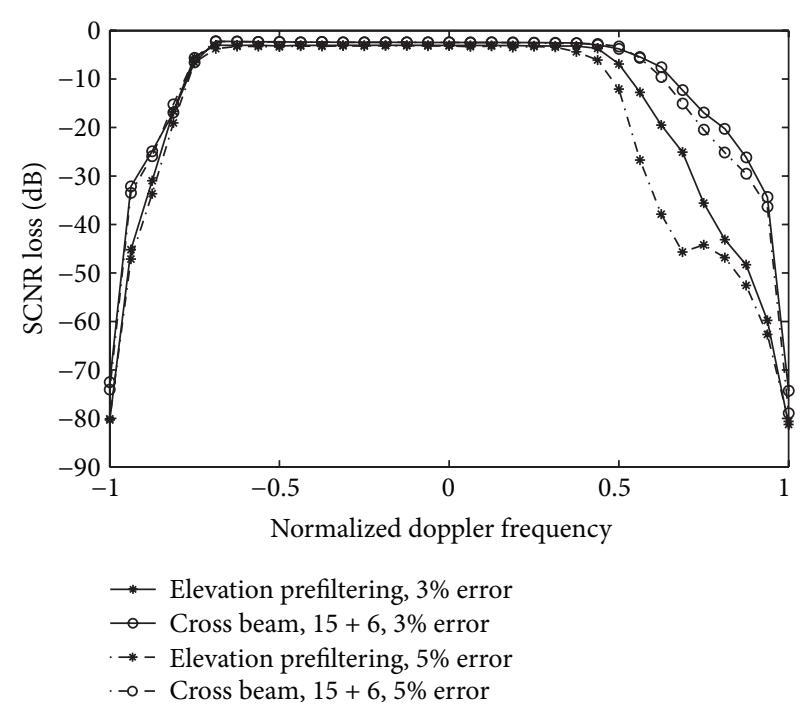

FIGURE 8: SCNR loss comparison of elevation prefiltering STAP and cross beam STAP with different errors.

Figure 8 shows the SCNR loss of the elevation prefiltering STAP method and cross beam STAP method when element errors exist. From Figure 8 we can see that the clutter suppression performance in short-range region of elevation prefiltering STAP method evidently degrades than the no error situation. However, the proposed cross beam STAP method's performance only slightly degrades and is significantly superior to the elevation prefiltering STAP method. Therefore, the cross beam STAP method has better robustness than the elevation prefiltering STAP method.

\section{Conclusions}

A new postbeam STAP method for nonstationary clutter suppression in airborne radar has been presented. Simulation 
results show that the short-range clutter suppression performance of cross beam STAP is superior to the existing 2D STAP, 3D STAP, and elevation prefiltering STAP methods. The computation load of the new method is close to the $2 \mathrm{D}$ and 3D STAP when the system DOF is chosen to be the same, and is larger than the elevation prefiltering STAP.

\section{Acknowledgment}

This work was supported in part by the National Nature Science Foundation of China under contract 60925005 and 61102169 .

\section{References}

[1] R. Klemm, Principles of Space-Time Adaptive Processing, IEE, London, UK, 2002.

[2] Y. L. Wang and Y. N. Peng, "Space-time adaptive processing for airborne radar with various array orientations," IEE Proceedings, vol. 144, no. 6, pp. 330-340, 1997.

[3] Y. Wang, Z. Bao, and Y. Peng, "STAP with medium PRF mode for non-side-looking airborne radar," IEEE Transactions on Aerospace and Electronic Systems, vol. 36, no. 2, pp. 609-620, 2004.

[4] O. Kreyenkamp and R. Klemm, "Doppler compensation in forward-looking STAP radar," IEE Proceedings, vol. 148, no. 5, pp. 253-258, 2001.

[5] B. Himed and A. Hajjari, "STAP with angle-doppler compensation for bistatic airborne radars," in Proceedings of the 2002 IEEE Radar Conference, pp. 22-25, Long Beach, Calif, USA, April 2002.

[6] W. L. Melvin and M. E. Davis, "Adaptive cancellation method for geometry-induced nonstationary bistatic clutter environments," IEEE Transactions on Aerospace and Electronic Systems, vol. 43, no. 2, pp. 651-672, 2007.

[7] P. M. Corbell and T. B. Hale, "3-dimensional STAP performance analysis using the cross-spectral metric," in Proceedings of the IEEE Radar Conference, pp. 610-615, Long Beach, Calif, USA, April 2004.

[8] X. Meng, T. Wang, J. Wu, and Z. Bao, "Short-range clutter suppression for airborne radar by utilizing prefiltering in elevation," IEEE Geoscience and Remote Sensing Letters, vol. 6, no. 2, pp. 268-272, 2009.

[9] Z. H. Zhang, W. C. Xie, and W. D. Hu, "Local degrees of freedom of airborne array radar clutter for STAP," IEEE Geoscience and Remote Sensing Letters, vol. 6, no. 1, pp. 97-101, 2009. 

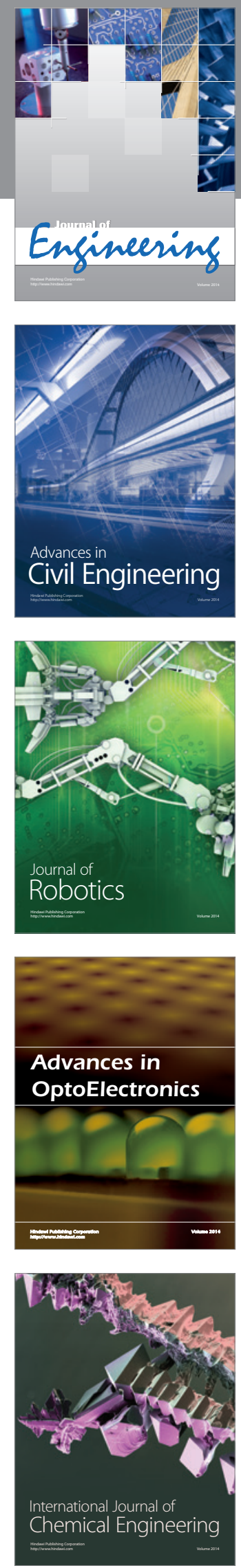

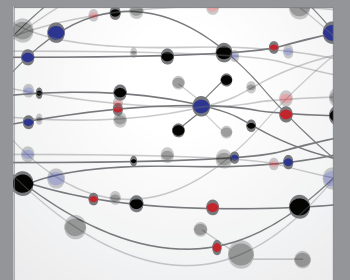

The Scientific World Journal
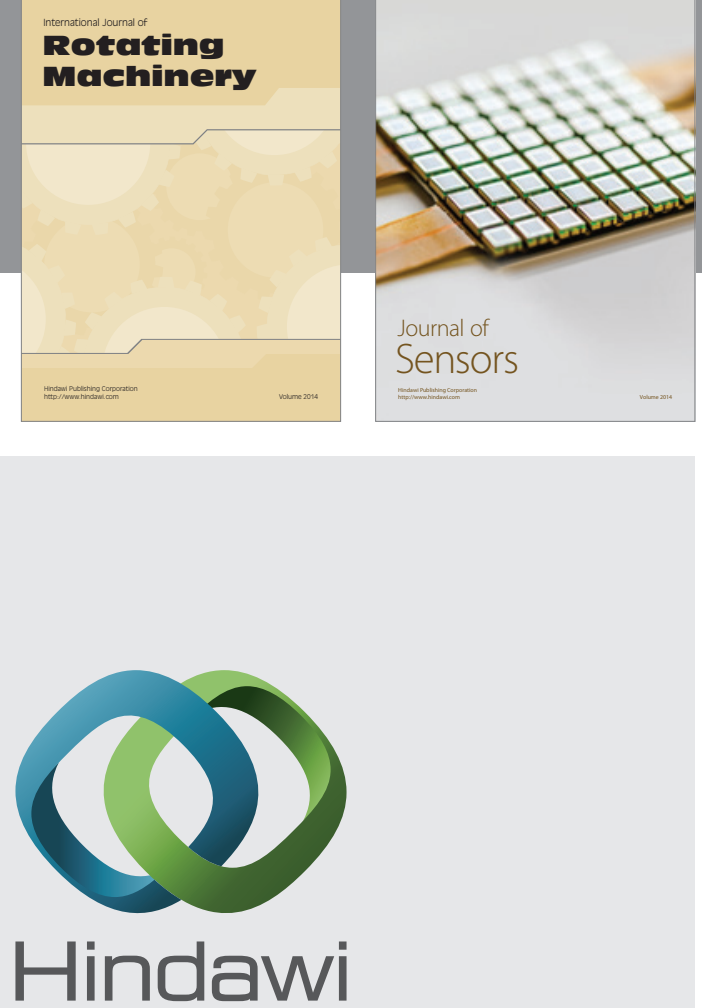

Submit your manuscripts at http://www.hindawi.com
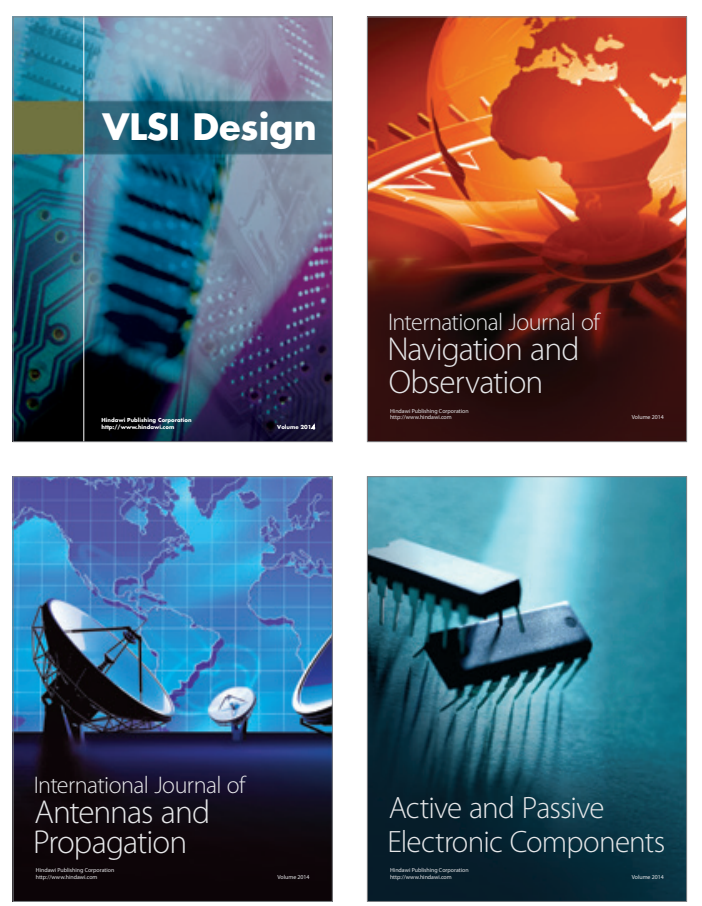
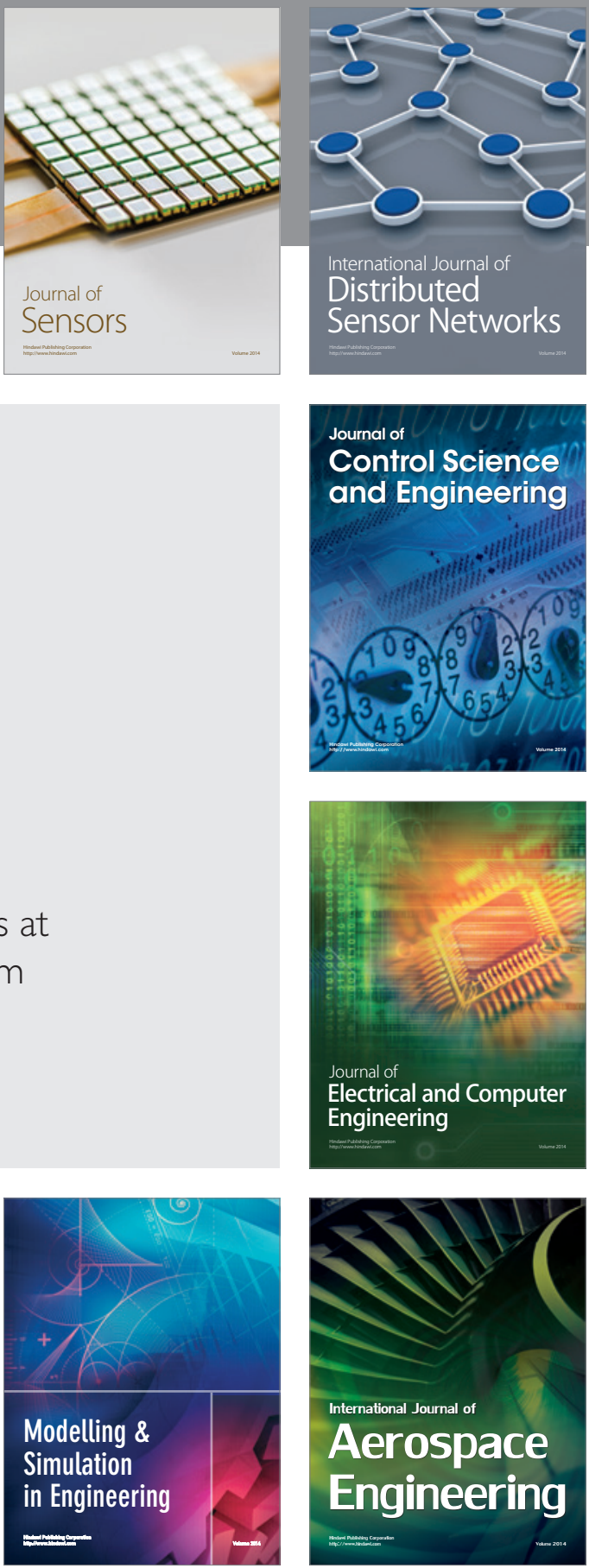

Journal of

Control Science

and Engineering
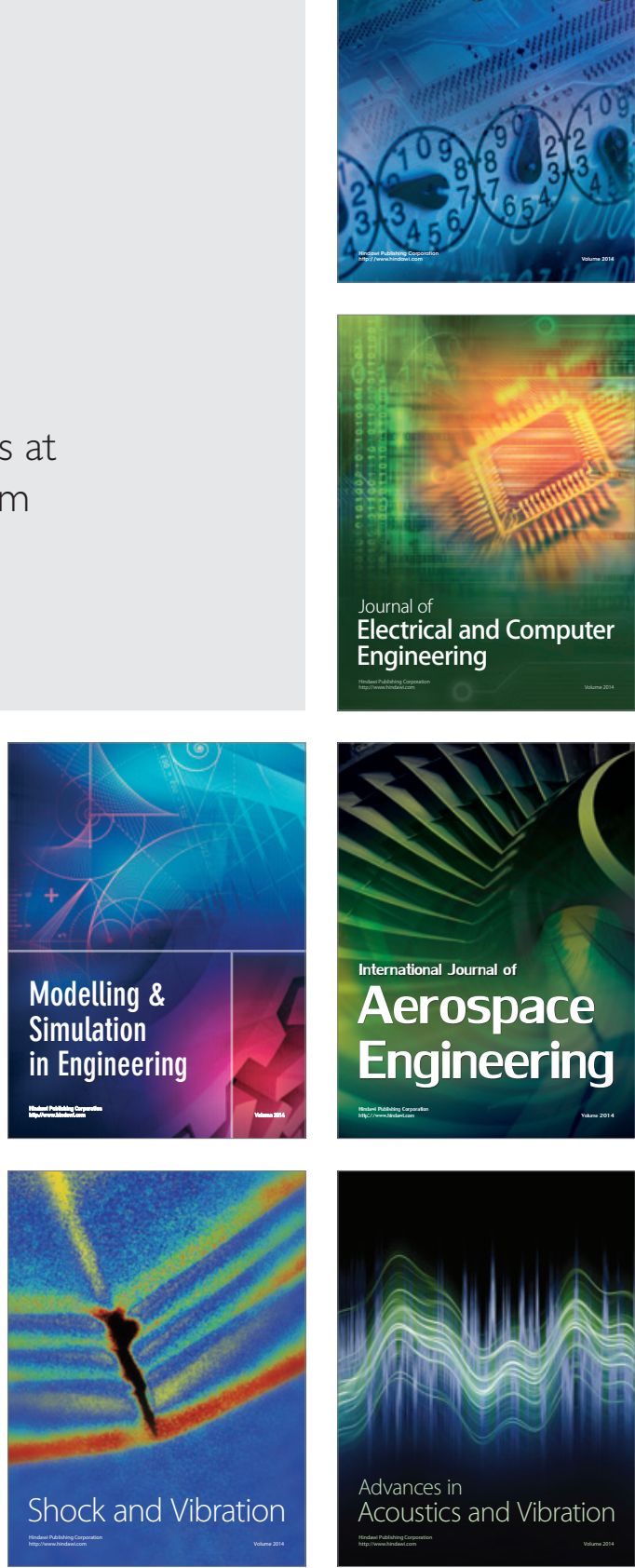